\title{
The charging of neutral dimethylamine and dimethylamine-sulfuric acid clusters using protonated acetone
}

\section{K. Ruusuvuori et al.}

Correspondence to: K. Ruusuvuori (kai.ruusuvuori@ helsinki.fi) 


\begin{tabular}{|c|c|c|c|c|}
\hline Cluster & $\begin{array}{c}\text { Delta E } \\
\text { (kcal/mol) }\end{array}$ & $\begin{array}{c}\text { Delta H } \\
\text { (kcal/mol) }\end{array}$ & $\begin{array}{c}\text { Delta G } \\
\text { (kcal/mol) }\end{array}$ & $\begin{array}{c}\text { Delta S } \\
\text { (kcal/mol) }\end{array}$ \\
\hline $\mathbf{H}^{+}(\mathbf{A c})_{1}(\mathrm{DMA})_{1}{ }^{*}$ & -24.33 & -23.18 & -15.11 & -27.08 \\
\hline $\mathbf{H}^{+}(\mathbf{D M A})_{1}{ }^{*}$ & - & - & - & - \\
\hline $\mathbf{H}^{+}(\mathbf{D M A})_{2}^{*, \mathbf{a}}$ & -26.33 & -25.68 & -16.89 & -29.45 \\
\hline $\mathbf{H}^{+}(\mathrm{SA})_{1}(\mathrm{DMA})_{2}{ }^{*}, \mathbf{b}$ & -65.60 & -61.47 & -44.58 & -56.64 \\
\hline $\mathbf{H}^{+}(\mathrm{SA})_{1}(\mathrm{DMA})_{3}{ }^{*, a}$ & -98.15 & -91.60 & -61.05 & -102.45 \\
\hline $\mathbf{H}^{+}(\mathbf{A c})_{2}(\mathrm{DMA})_{1}{ }^{*}$ & -43.92 & -41.30 & -25.51 & -52.97 \\
\hline$(\mathrm{SA})_{1}(\mathrm{DMA})_{1}{ }^{\mathrm{c}}$ & -27.22 & -24.65 & -15.40 & -31.01 \\
\hline$(\mathrm{Ac})_{1}(\mathrm{SA})_{1}$ & -15.67 & -14.27 & -4.03 & -34.36 \\
\hline$(\mathrm{Ac})_{1}(\mathrm{DMA})_{1}$ & -6.47 & -4.95 & 3.92 & -29.74 \\
\hline $\mathbf{H}^{+}(\mathbf{A c})_{1}$ & 30.09 & 28.11 & 28.13 & -0.07 \\
\hline $\mathbf{H}^{+}(\mathbf{A c})_{2}$ & -4.35 & -6.84 & 3.07 & -33.22 \\
\hline $\mathbf{H}^{+}(\mathbf{A c})_{3}$ & -18.21 & -18.03 & 0.26 & -61.36 \\
\hline $\mathbf{H}^{+}(\mathbf{D M A})_{3^{\mathrm{a}}}$ & -46.41 & -43.77 & -25.80 & -60.28 \\
\hline $\mathbf{H}^{+}(\mathrm{SA})_{1}(\mathrm{DMA})_{1}{ }^{\mathrm{b}}$ & -19.29 & -18.19 & -10.83 & -24.68 \\
\hline $\mathbf{H}^{+}(\mathbf{S A})_{1}(\mathbf{A c})_{2}$ & -19.54 & -20.25 & -0.79 & -65.27 \\
\hline $\mathbf{H}^{+}(\mathrm{Ac})_{1}(\mathrm{DMA})_{2}$ & -46.16 & -43.68 & -25.71 & -60.28 \\
\hline $\mathrm{H}^{+}(\mathrm{SA})_{1}(\mathrm{Ac})_{1}(\mathrm{DMA})_{1}$ & -43.55 & -42.40 & -25.75 & -55.86 \\
\hline $\mathrm{H}^{+}(\mathrm{SA})_{1}(\mathrm{Ac})_{2}(\mathrm{DMA})_{1}$ & -64.66 & -61.57 & -33.20 & -95.14 \\
\hline
\end{tabular}

*Cluster types listed in Figure $3 \mathrm{c}$ in the main article.

a)Previously published in Almeida et al. (2013) at a different temperature.

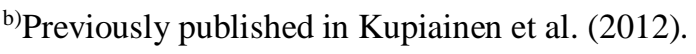

${ }^{c}$ Published previously by Ortega et al. (2012).

Table S1. The Gibbs free energies of formation for the allowed cluster types. For these energies, $\mathrm{H}^{+}(\mathrm{DMA})_{1}$ is considered a monomer, which is why no energies are listed for its formation. This also means that the Delta $\mathrm{G}$ for e.g. $\mathrm{H}^{+}(\mathrm{DMA})_{2}$ is calculated from the Gibbs 
free energies of $\mathrm{H}^{+}(\mathrm{DMA})_{1}$ and $(\mathrm{DMA})_{1}$. The energies of $\mathrm{H}^{+}(\mathrm{Ac})_{1}$ are for the proton transfer reaction $\mathrm{H}^{+}(\mathrm{DMA})_{1}+(\mathrm{Ac})_{1} \rightarrow(\mathrm{DMA})_{1}+\mathrm{H}^{+}(\mathrm{Ac})_{1}$. Thus, the energies reported for the formation of clusters containing acetone also include this proton transfer energy. 


\begin{tabular}{ccc}
\hline Cluster & Dipole moment (D) & Polarizability $\left(\AA^{\mathbf{3}}\right)$ \\
\hline$(\mathbf{S A})_{\mathbf{1}}$ & $2.96^{\mathrm{a}}$ & $6.2^{\mathrm{b}}$ \\
\hline$(\mathbf{D M A})_{\mathbf{1}}$ & $1.01^{\mathrm{c}}$ & $6.37^{\mathrm{c}}$ \\
\hline$(\mathbf{A c})_{\mathbf{1}}$ & $2.88^{\mathrm{c}}$ & $6.33^{\mathrm{c}}$ \\
\hline$(\mathbf{S A})_{\mathbf{1}}(\mathbf{D M A})_{\mathbf{1}}$ & $8.76^{\mathrm{d}}$ & $9.37^{\mathrm{d}}$ \\
\hline$(\mathbf{A c})_{\mathbf{1}}(\mathbf{S A})_{\mathbf{1}}$ & $4.72^{\mathrm{e}}$ & $10.10^{\mathrm{e}}$ \\
\hline$(\mathbf{A c})_{\mathbf{1}}(\mathbf{D M A})_{\mathbf{1}}$ & $2.62^{\mathrm{e}}$ & $10.45^{\mathrm{e}}$ \\
\hline
\end{tabular}

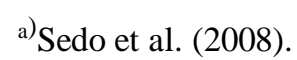

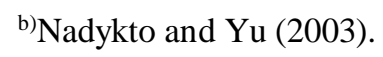

${ }^{c}$ CRC Handbook of Chemistry and Physics (Lide, 2010).

d)Previously published in Almeida et al. (2013).

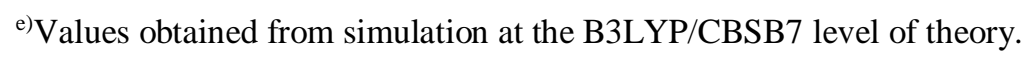

Table S2. Dipole moments and polarizabilities for the neutral clusters and monomers. 

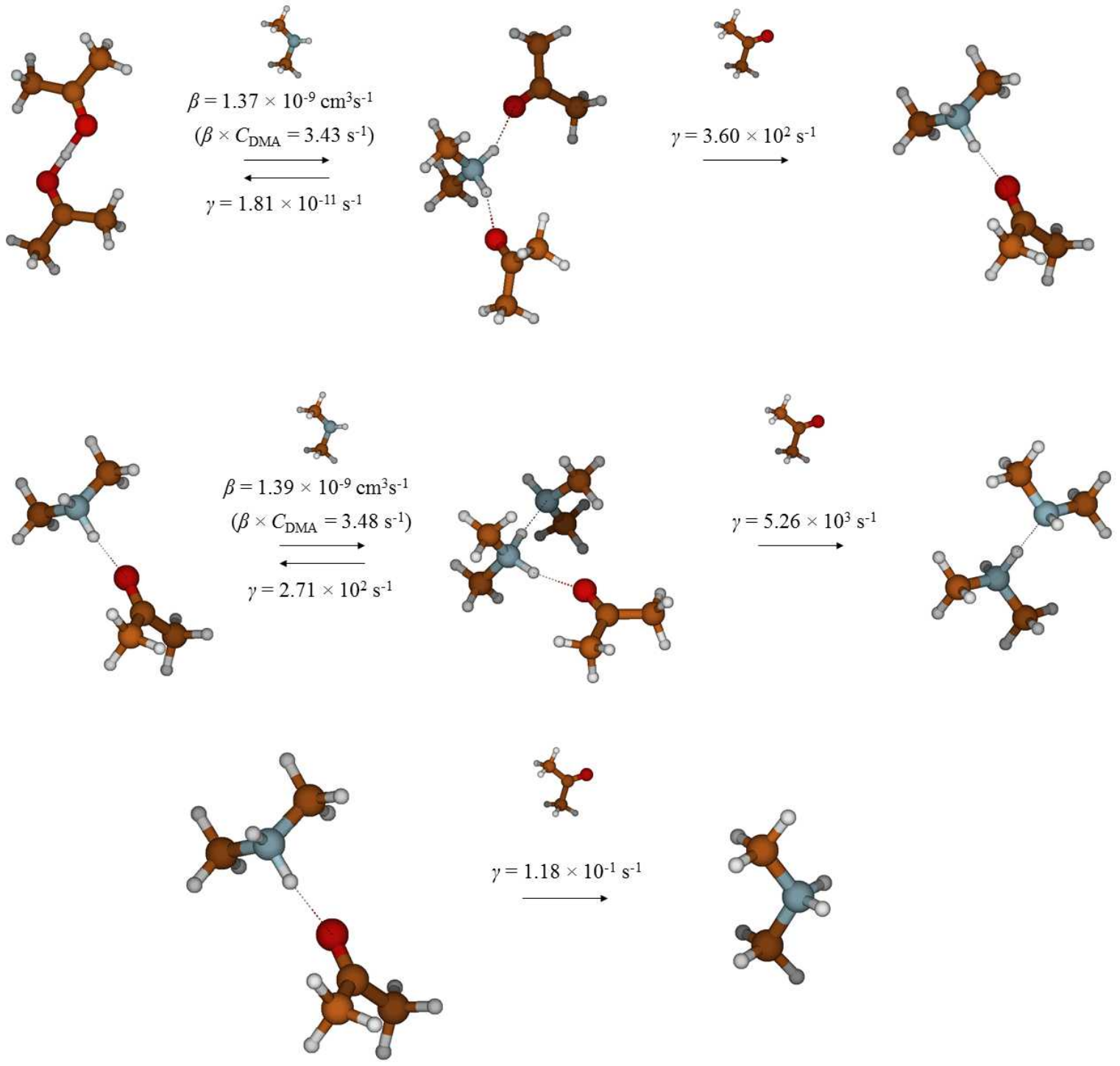

Figure S1. The dominant reaction paths leading to the formation of the cluster types (from top to bottom $) \mathrm{H}^{+}(\mathrm{Ac})_{1}(\mathrm{DMA})_{1}, \mathrm{H}^{+}(\mathrm{DMA})_{2}$ and $\mathrm{H}^{+}(\mathrm{DMA})_{1}$. In the figure, $\gamma$ is the evaporation coefficient and $\beta$ is the collision coefficient. The values in parentheses are example values calculated assuming $[\mathrm{DMA}]=100$ ppt. Collisions with neutral acetone monomers are not included, since the initial neutral acetone monomer concentration was assumed to be zero in the simulations. 


\section{References}

Almeida, J., Schobesberger, S., Kürten, A., Ortega, I. K., Kupiainen-Määttä, O., Praplan, A. P., Adamov, A., Amorim, A., Bianchi, F., Breitenlechner, M., David, A., Dommen, J., Donahue, N. M., Downard, A., Dunne, E., Duplissy, J., Ehrhart, S., Flagan, R. C., Franchin, A., Guida, R., Hakala, J., Hansel, A., Heinritzi, M., Henschel, H., Jokinen, T., Junninen, H., Kajos, M., Kangasluoma, J., Keskinen, H., Kupc, A., Kurtén, T., Kvashin, A. N., Laaksonen, A., Lehtipalo, K., Leiminger, M., Leppä, J., Loukonen, V., Makhmutov, V., Mathot, S., McGrath, M. J., Nieminen, T., Olenius, T., Onnela, A., Petäjä, T., Riccobono, F., Riipinen, I., Rissanen, M., Rondo, L., Ruuskanen, T., Santos, F. D., Sarnela, N., Schallhart, S., Schnitzhofer, R., Seinfeld, J. H., Simon, M., Sipilä, M., Stozhkov, Y., Stratmann, F.,Tomé, A., Tröstl, J., Tsagkogeorgas, G., Vaattovaara, P., Viisanen, Y., Virtanen, A., Vrtala, A., Wagner, P. E., Weingartner, E., Wex, H., Williamson, C., Wimmer, D., Ye, P., Yli-Juuti, T., Carslaw, K. S., Kulmala, M., Curtius, J., Baltensperger, U., Worsnop, D. R., Vehkamäki, H., and Kirkby, J.: Molecular understanding of sulphuric acid-amine particle nucleation in the atmosphere, Nature, 502, 359-363, 2013.

Lide, D. (Ed.): CRC Handbook of Chemistry and Physics, 91th Edition, CRC Press/Taylor and Francis, Boca Raton, FL, 2010.

Nadykto, A., Yu, F., Jakovleva, M., Herb, J., and Xu, Y.: Amines in the Earth's Atmosphere: A Density Functional Theory Study of the Thermochemistry of Pre-Nucleation Clusters, Entropy, 13, 554-569, 2011.

Ortega, I. K., Kupiainen, O., Kurtén, T., Olenius, T., Wilkman, O., McGrath, M. J., Loukonen, V., and Vehkamäki, H., From quantum chemical formation free energies to evaporation rates, Atmos. Chem. Phys. 12, 225-235, 2012.

Sedo, G., Schultz, J., and Leopold, K. R.: Electric dipole moment of sulfuric acid from Fourier transform microwave spectroscopy, J. Mol. Spectrosc., 251, 4-8, doi:10.1016/j.jms.2007.09.016, 2008. 
Cartesian coordinates for the most stable structure of each cluster, optimized at the B3LYP/CBSB7 level.

Neutral:

$\left(\mathrm{H}_{2} \mathrm{SO}_{4}\right)_{1}$

\begin{tabular}{llll} 
S & 0.000000 & 0.000000 & 0.165252 \\
O & 0.000000 & 1.262812 & 0.826069 \\
O & 0.000000 & -1.262812 & 0.826069 \\
O & 1.247362 & -0.047261 & -0.851640 \\
O & -1.247362 & 0.047261 & -0.851640 \\
H & -1.456139 & -0.860946 & -1.117451 \\
H & 1.456139 & 0.860946 & -1.117451 \\
\hline
\end{tabular}

$\left(\left(\mathrm{CH}_{3}\right)_{2} \mathrm{NH}\right)_{1}$

\begin{tabular}{|c|c|c|c|}
\hline $\mathrm{N}$ & 0.000000 & 0.563912 & -0.148594 \\
\hline $\mathrm{C}$ & 1.215453 & -0.222523 & 0.020305 \\
\hline $\mathrm{C}$ & -1.215453 & -0.222523 & 0.020305 \\
\hline $\mathrm{H}$ & 0.000001 & 1.329114 & 0.517154 \\
\hline $\mathrm{H}$ & 1.282780 & -0.965827 & -0.780467 \\
\hline $\mathrm{H}$ & 2.089686 & 0.427944 & -0.061159 \\
\hline $\mathrm{H}$ & 1.275377 & -0.765224 & 0.981298 \\
\hline $\mathrm{H}$ & -1.282775 & -0.965832 & -0.780462 \\
\hline $\mathrm{H}$ & -1.275381 & -0.765218 & 0.981301 \\
\hline $\mathrm{H}$ & -2.089687 & 0.427943 & -0.061167 \\
\hline
\end{tabular}

$\left(\mathrm{C}_{3} \mathrm{H}_{6} \mathrm{O}\right)_{1}$
C $\quad-0.000070$
0.186391
$-0.000129$ 


\begin{tabular}{lrrr}
$\mathrm{C}$ & 1.291451 & -0.613508 & -0.001609 \\
$\mathrm{C}$ & -1.291539 & -0.613340 & 0.001621 \\
$\mathrm{O}$ & 0.000129 & 1.395491 & 0.000031 \\
$\mathrm{H}$ & -1.376888 & -1.187461 & -0.926959 \\
$\mathrm{H}$ & -1.301428 & -1.334910 & 0.824231 \\
$\mathrm{H}$ & -2.141469 & 0.061889 & 0.089311 \\
$\mathrm{H}$ & 2.141221 & 0.061763 & -0.091149 \\
$\mathrm{H}$ & 1.377612 & -1.185888 & 0.927951 \\
$\mathrm{H}$ & 1.300862 & -1.336578 & -0.822919 \\
\hline
\end{tabular}

$\left(\mathrm{H}_{2} \mathrm{SO}_{4}\right)_{1} \cdot\left(\left(\mathrm{CH}_{3}\right)_{2} \mathrm{NH}\right)_{1}$

$\begin{array}{lrrr}\text { H } & 1.202657 & -0.116413 & 0.805505 \\ \text { S } & -1.285655 & -0.159813 & 0.012780 \\ \text { O } & -1.799961 & 1.405256 & 0.038106 \\ \text { O } & -0.439059 & -0.259155 & -1.202714 \\ \text { O } & -2.469017 & -0.979113 & -0.010431 \\ \text { O } & -0.456443 & -0.215855 & 1.230045 \\ \text { H } & -2.600799 & 1.438544 & -0.501892 \\ \text { N } & 1.882601 & -0.026869 & 0.007871 \\ \text { H } & 1.159416 & -0.130108 & -0.760163 \\ \text { C } & 2.856478 & -1.140467 & -0.007379 \\ \text { H } & 3.503993 & -1.077114 & 0.867623 \\ \text { H } & 3.459129 & -1.088258 & -0.914467 \\ \text { H } & 2.305790 & -2.079796 & 0.011578 \\ \text { C } & 2.459827 & 1.337301 & -0.016143 \\ \text { H } & 3.093965 & 1.484705 & 0.858389 \\ \text { H } & 1.639041 & 2.052859 & -0.000146 \\ \text { H } & 3.047081 & 1.470607 & -0.924910 \\ ----------------------------------------------\end{array}$




$\begin{array}{llll}\mathrm{C} & -3.769214 & -0.543568 & 0.404276 \\ \mathrm{C} & -2.444458 & -0.070755 & -0.131568 \\ \mathrm{C} & -2.146690 & 1.406874 & -0.087712 \\ \mathrm{H} & -1.448792 & 1.583855 & 0.737248 \\ \mathrm{H} & -3.042932 & 2.008381 & 0.064922 \\ \mathrm{H} & -1.638421 & 1.714004 & -1.003357 \\ \mathrm{H} & -0.121198 & -0.466957 & -0.955708 \\ \mathrm{O} & -1.634978 & -0.876314 & -0.570012 \\ \mathrm{~S} & 1.664215 & 0.121459 & 0.090762 \\ \mathrm{O} & 0.819400 & 0.648119 & 1.120021 \\ \mathrm{O} & 2.855332 & 0.778661 & -0.346400 \\ \mathrm{O} & 0.814870 & -0.183492 & -1.199870 \\ \mathrm{O} & 2.058371 & -1.358446 & 0.610673 \\ \mathrm{H} & -3.915233 & -0.167014 & 1.421569 \\ \mathrm{H} & -3.816180 & -1.630985 & 0.394674 \\ \mathrm{H} & -4.579915 & -0.129514 & -0.204481 \\ \mathrm{H} & 2.793439 & -1.678646 & 0.067668 \\ --------------------\end{array}$

$\left(\mathrm{C}_{3} \mathrm{H}_{6} \mathrm{O}\right)_{1} \cdot\left(\left(\mathrm{CH}_{3}\right)_{2} \mathrm{NH}\right)_{1}$

$\begin{array}{llll}\text { C } & -1.498973 & -0.014566 & 0.249280 \\ \text { C } & -1.780729 & 1.278894 & -0.490325 \\ \text { C } & -1.711181 & -1.295261 & -0.534004 \\ \text { O } & -1.106138 & -0.023502 & 1.395537 \\ \mathrm{H} & -1.629015 & -2.154679 & 0.130249 \\ \mathrm{H} & -2.682051 & -1.300531 & -1.037537 \\ \mathrm{H} & -0.936082 & -1.355425 & -1.303900 \\ \mathrm{H} & -1.634885 & 2.127169 & 0.177088 \\ \mathrm{H} & -1.097722 & 1.357970 & -1.341131 \\ \mathrm{H} & -2.801402 & 1.287914 & -0.884778 \\ \mathrm{H} & 1.827235 & -2.083434 & -0.466761\end{array}$




$\begin{array}{lrrr}\mathrm{N} & 1.664125 & 0.004876 & -0.625817 \\ \mathrm{C} & 1.944072 & 1.232890 & 0.116522 \\ \mathrm{C} & 1.982246 & -1.193663 & 0.148793 \\ \mathrm{H} & 2.210667 & 0.002293 & -1.481038 \\ \mathrm{H} & 1.269228 & 1.293310 & 0.974065 \\ \mathrm{H} & 2.979360 & 1.297201 & 0.493953 \\ \mathrm{H} & 1.306503 & -1.254681 & 1.005616 \\ \mathrm{H} & 3.017864 & -1.213843 & 0.530112 \\ \mathrm{H} & 1.757915 & 2.100847 & -0.521111\end{array}$

\section{Positively charged:}

$\begin{array}{crrr}\left(\mathrm{C}_{3} \mathrm{H}_{7} \mathrm{O}\right)_{1}{ }^{+} & & \\ \mathrm{C} & -0.005068 & 0.060159 & -0.000139 \\ \mathrm{C} & 1.229521 & -0.745071 & 0.006681 \\ \mathrm{C} & -1.346019 & -0.540740 & -0.008219 \\ \mathrm{O} & 0.029653 & 1.337259 & -0.005069 \\ \mathrm{H} & 2.115492 & -0.177272 & 0.295791 \\ \mathrm{H} & 1.110191 & -1.624231 & 0.644851 \\ \mathrm{H} & 1.373141 & -1.128062 & -1.015209 \\ \mathrm{H} & -1.567589 & -0.843400 & 1.027691 \\ \mathrm{H} & -2.105908 & 0.169341 & -0.330709 \\ \mathrm{H} & -1.361699 & -1.456100 & -0.604029 \\ \mathrm{H} & 0.928543 & 1.715559 & 0.032231\end{array}$

$\left(\mathrm{C}_{3} \mathrm{H}_{6} \mathrm{O}\right)_{1} \cdot\left(\mathrm{C}_{3} \mathrm{H}_{7} \mathrm{O}\right)_{1}{ }^{+}$

$\begin{array}{llll}\mathrm{C} & 2.140424 & -0.009353 & -0.004469 \\ \mathrm{C} & 2.166367 & 1.480109 & -0.014831 \\ \mathrm{C} & 3.413255 & -0.774738 & 0.011167 \\ \mathrm{O} & 1.068649 & -0.653441 & -0.012306\end{array}$




$\begin{array}{lrrr}\mathrm{H} & 1.199011 & 1.918939 & 0.223726 \\ \mathrm{H} & 2.936699 & 1.851706 & 0.664705 \\ \mathrm{H} & 2.462335 & 1.801517 & -1.021725 \\ \mathrm{H} & 3.828395 & -0.718527 & 1.025951 \\ \mathrm{H} & 3.247513 & -1.819231 & -0.245211 \\ \mathrm{H} & 4.151947 & -0.317670 & -0.651326 \\ \mathrm{H} & 0.070818 & -0.081984 & 0.006771 \\ \mathrm{C} & -2.131935 & 0.008348 & 0.006144 \\ \mathrm{C} & -3.357814 & 0.855819 & 0.006648 \\ \mathrm{C} & -2.273057 & -1.479542 & -0.020677 \\ \mathrm{O} & -1.020383 & 0.564051 & 0.032192 \\ \mathrm{H} & -2.610034 & -1.806742 & 0.970550 \\ \mathrm{H} & -3.052660 & -1.777837 & -0.725383 \\ \mathrm{H} & -1.336728 & -1.982885 & -0.257388 \\ \mathrm{H} & -3.120615 & 1.885383 & 0.266992 \\ \mathrm{H} & -3.791524 & 0.831955 & -1.000799 \\ \mathrm{H} & -4.114735 & 0.446639 & 0.680162 \\ ----------------------------------------------------\end{array}$

$\left(\mathrm{C}_{3} \mathrm{H}_{6} \mathrm{O}\right)_{2} \cdot\left(\mathrm{C}_{3} \mathrm{H}_{7} \mathrm{O}\right)_{1}{ }^{+}$

$\begin{array}{llll}\mathrm{C} & -3.065048 & 1.013812 & 0.009180 \\ \mathrm{C} & -3.273373 & 1.484196 & -1.410717 \\ \mathrm{C} & -3.915911 & 1.655590 & 1.077560 \\ \mathrm{O} & -2.244405 & 0.151800 & 0.280212 \\ \mathrm{H} & -3.816828 & 1.119629 & 2.020171 \\ \mathrm{H} & -3.593907 & 2.693140 & 1.218939 \\ \mathrm{H} & -4.965354 & 1.692523 & 0.773248 \\ \mathrm{H} & -4.247237 & 1.131851 & -1.767629 \\ \mathrm{H} & -3.297990 & 2.575923 & -1.463096 \\ \mathrm{H} & -2.492128 & 1.094335 & -2.061681 \\ \mathrm{C} & 3.318835 & 0.699358 & 0.033629 \\ \mathrm{C} & 3.569592 & 0.794642 & -1.442863 \\ \mathrm{C} & 4.270139 & 1.385730 & 0.963417\end{array}$




$\begin{array}{rrrr}\mathrm{O} & 2.362410 & 0.070492 & 0.494322 \\ \mathrm{H} & 4.097499 & 1.074414 & 1.991705 \\ \mathrm{H} & 5.305849 & 1.194308 & 0.671038 \\ \mathrm{H} & 4.118254 & 2.468455 & 0.884170 \\ \mathrm{H} & 2.705862 & 0.475094 & -2.024854 \\ \mathrm{H} & 3.857682 & 1.810364 & -1.723157 \\ \mathrm{H} & 4.420726 & 0.149474 & -1.690348 \\ \mathrm{C} & -0.215367 & -1.822646 & -0.004154 \\ \mathrm{C} & -1.221344 & -2.667680 & -0.685877 \\ \mathrm{C} & -0.175247 & -1.735514 & 1.472873 \\ \mathrm{O} & 0.609430 & -1.207635 & -0.733564 \\ \mathrm{H} & -2.191834 & -2.559586 & -0.200419 \\ \mathrm{H} & -1.283195 & -2.428211 & -1.745122 \\ \mathrm{H} & -0.913059 & -3.715013 & -0.570031 \\ \mathrm{H} & -0.407796 & -2.702845 & 1.921863 \\ \mathrm{H} & 0.768958 & -1.342929 & 1.846169 \\ \mathrm{H} & -0.986863 & -1.045831 & 1.741592 \\ \mathrm{H} & 1.348228 & -0.647275 & -0.228610 \\ ----------------------------------------------------\end{array}$

$\left(\left(\mathrm{CH}_{3}\right)_{2} \mathrm{NH}_{2}\right)_{1}{ }^{+}$

$\begin{array}{llrl}\text { N } & 0.000000 & 0.000000 & 0.540852 \\ \mathrm{C} & 0.000000 & 1.267957 & -0.276150 \\ \mathrm{C} & 0.000000 & -1.267957 & -0.276150 \\ \mathrm{H} & 0.816156 & -0.000007 & 1.159149 \\ \mathrm{H} & -0.816156 & 0.000007 & 1.159149 \\ \mathrm{H} & 0.893134 & 1.276122 & -0.897827 \\ \mathrm{H} & -0.893131 & 1.276124 & -0.897834 \\ \mathrm{H} & 0.893131 & -1.276124 & -0.897834 \\ \mathrm{H} & -0.893134 & -1.276122 & -0.897827 \\ \mathrm{H} & -0.000001 & 2.120753 & 0.400431 \\ \mathrm{H} & 0.000001 & -2.120753 & 0.400431\end{array}$




\begin{tabular}{|c|c|c|c|}
\hline $\mathrm{C}$ & 1.800148 & -0.026540 & 0.009395 \\
\hline $\mathrm{C}$ & 2.379530 & 1.213149 & -0.615568 \\
\hline $\mathrm{C}$ & 2.757494 & -1.041415 & 0.566484 \\
\hline $\mathrm{O}$ & 0.588449 & -0.215479 & 0.073074 \\
\hline $\mathrm{H}$ & 3.275223 & -1.534485 & -0.264095 \\
\hline $\mathrm{H}$ & 3.529494 & -0.559154 & 1.171885 \\
\hline $\mathrm{H}$ & 2.226511 & -1.789703 & 1.151833 \\
\hline $\mathrm{H}$ & 1.622739 & 1.773633 & -1.163104 \\
\hline $\mathrm{H}$ & 2.790855 & 1.848651 & 0.176817 \\
\hline $\mathrm{H}$ & 3.212929 & 0.964047 & -1.277135 \\
\hline $\mathrm{N}$ & -2.013161 & 0.326303 & -0.299995 \\
\hline $\mathrm{C}$ & -2.511539 & 0.702411 & 1.062176 \\
\hline $\mathrm{C}$ & -2.487417 & -1.013163 & -0.776706 \\
\hline $\mathrm{H}$ & -2.292748 & 1.042801 & -0.971900 \\
\hline $\mathrm{H}$ & -0.963257 & 0.306311 & -0.273686 \\
\hline $\mathrm{H}$ & -3.600217 & 0.716132 & 1.058328 \\
\hline $\mathrm{H}$ & -2.146435 & -0.035556 & 1.773755 \\
\hline $\mathrm{H}$ & -3.575896 & -1.024769 & -0.799172 \\
\hline $\mathrm{H}$ & -2.113582 & -1.769052 & -0.089341 \\
\hline $\mathrm{H}$ & -2.123603 & 1.686575 & 1.319369 \\
\hline $\mathrm{H}$ & -2.086777 & -1.192370 & -1.772869 \\
\hline
\end{tabular}

$\left(\mathrm{C}_{3} \mathrm{H}_{6} \mathrm{O}\right)_{2} \cdot\left(\left(\mathrm{CH}_{3}\right)_{2} \mathrm{NH}_{2}\right)_{1}{ }^{+}$

$\begin{array}{llll}\mathrm{C} & 3.528822 & -0.606586 & -0.077946 \\ \mathrm{C} & 3.342715 & -2.100705 & -0.136849 \\ \mathrm{C} & 4.937913 & -0.079474 & -0.119990 \\ \mathrm{O} & 2.576668 & 0.159511 & -0.003260 \\ \mathrm{H} & 4.938081 & 1.001224 & -0.249918 \\ \mathrm{H} & 5.442150 & -0.329818 & 0.819951 \\ \mathrm{H} & 5.510168 & -0.559051 & -0.918662\end{array}$




\begin{tabular}{lrrr}
$\mathrm{H}$ & 3.569127 & -2.446091 & -1.151813 \\
$\mathrm{H}$ & 4.042999 & -2.610147 & 0.529721 \\
$\mathrm{H}$ & 2.319031 & -2.377420 & 0.111997 \\
$\mathrm{~N}$ & 0.004971 & 1.116142 & 0.124072 \\
$\mathrm{C}$ & -0.008009 & 2.003563 & -1.076744 \\
$\mathrm{H}$ & 0.038467 & 1.856906 & 1.419553 \\
$\mathrm{H}$ & -0.848811 & 0.527815 & 0.098765 \\
$\mathrm{H}$ & 0.846783 & 0.512439 & 0.072622 \\
$\mathrm{H}$ & -0.895158 & 2.632942 & -1.041754 \\
$\mathrm{H}$ & -0.894503 & 2.612038 & -1.076563 \\
$\mathrm{H}$ & 0.942535 & 2.461856 & 1.457744 \\
$\mathrm{H}$ & -0.034590 & 1.381537 & -1.969718 \\
$\mathrm{H}$ & 0.043308 & 1.136371 & 2.235850 \\
$\mathrm{C}$ & -3.535551 & -0.585047 & -0.047553 \\
$\mathrm{C}$ & -4.892712 & -0.106090 & -0.488609 \\
$\mathrm{C}$ & -3.428164 & -2.000284 & 0.458378 \\
$\mathrm{O}$ & -2.563413 & 0.157318 & -0.099566 \\
$\mathrm{H}$ & -3.925085 & -2.068488 & 1.432411 \\
$\mathrm{H}$ & -3.949561 & -2.693558 & -0.206595 \\
$\mathrm{H}$ & -2.386419 & -2.298625 & 0.568401 \\
$\mathrm{H}$ & -5.657113 & -0.364803 & 0.248928 \\
$\mathrm{H}$ & -4.881066 & 0.968496 & -0.661529 \\
----------------------------------------------------- \\
\hline
\end{tabular}

$\left(\mathrm{H}_{2} \mathrm{SO}_{4}\right)_{1} \cdot\left(\mathrm{C}_{3} \mathrm{H}_{6} \mathrm{O}\right)_{1} \cdot\left(\mathrm{C}_{3} \mathrm{H}_{7} \mathrm{O}\right)_{1}{ }^{+}$

$\begin{array}{llll}\text { C } & -2.664430 & -0.958104 & -1.489532 \\ \text { C } & -2.697962 & -0.705493 & -0.016346 \\ \text { C } & -3.360945 & -1.705798 & 0.871306 \\ \text { C } & -0.108488 & 2.630624 & 1.443568 \\ \text { C } & -0.000853 & 2.560728 & -0.037627 \\ \text { C } & 1.022169 & 3.364663 & -0.749246\end{array}$




\begin{tabular}{lrrr}
$\mathrm{O}$ & -0.761081 & 1.843365 & -0.728613 \\
$\mathrm{O}$ & -2.196680 & 0.307711 & 0.492005 \\
$\mathrm{~S}$ & 1.618536 & -1.090483 & 0.030702 \\
$\mathrm{O}$ & 1.805777 & 0.268056 & 0.442803 \\
$\mathrm{O}$ & 0.348847 & -1.597587 & -0.383416 \\
$\mathrm{O}$ & 2.107454 & -2.073162 & 1.186000 \\
$\mathrm{O}$ & 2.698595 & -1.321933 & -1.119277 \\
$\mathrm{H}$ & -2.370203 & -1.991331 & -1.685969 \\
$\mathrm{H}$ & -1.996020 & -0.275324 & -2.010470 \\
$\mathrm{H}$ & -3.681356 & -0.837571 & -1.880864 \\
$\mathrm{H}$ & 2.533563 & -2.168142 & -1.566726 \\
$\mathrm{H}$ & -3.575100 & -1.276362 & 1.848250 \\
$\mathrm{H}$ & -2.666675 & -2.545694 & 0.997796 \\
$\mathrm{H}$ & -4.266977 & -2.107819 & 0.413491 \\
$\mathrm{H}$ & 0.887606 & 2.582721 & 1.887670 \\
$\mathrm{H}$ & -0.748529 & 1.852703 & 1.854355 \\
$\mathrm{H}$ & -0.523791 & 3.613349 & 1.700274 \\
$\mathrm{H}$ & 2.890490 & -1.706602 & 1.628687 \\
$\mathrm{H}$ & 0.773710 & 3.473679 & -1.803449 \\
$\mathrm{H}$ & 1.966759 & 2.811928 & -0.664151 \\
$\mathrm{H}$ & 1.171198 & 4.335020 & -0.272891 \\
$\mathrm{H}$ & -1.451514 & 1.155859 & -0.185986 \\
--------------------------------------------------- \\
\hline
\end{tabular}

$\left(\mathrm{C}_{3} \mathrm{H}_{6} \mathrm{O}\right)_{1} \cdot\left(\left(\mathrm{CH}_{3}\right)_{2} \mathrm{NH}\right) \cdot\left(\left(\mathrm{CH}_{3}\right)_{2} \mathrm{NH}_{2}\right)_{1}{ }^{+}$

$\begin{array}{llll}\mathrm{C} & 2.594692 & 0.320308 & -0.019190 \\ \mathrm{C} & 3.197555 & 1.699326 & -0.010824 \\ \mathrm{C} & 3.544579 & -0.848747 & -0.058699 \\ \mathrm{O} & 1.379637 & 0.166011 & 0.003357 \\ \mathrm{H} & 3.029162 & -1.783579 & 0.158107 \\ \mathrm{H} & 3.988993 & -0.910125 & -1.058428 \\ \mathrm{H} & 4.371373 & -0.705692 & 0.641475 \\ \mathrm{H} & 2.435154 & 2.454351 & -0.194098\end{array}$




$\begin{array}{lrrr}\mathrm{H} & 3.653769 & 1.880886 & 0.968875 \\ \mathrm{H} & 3.999750 & 1.781388 & -0.748755 \\ \mathrm{~N} & -2.475860 & 0.975931 & 0.050587 \\ \mathrm{C} & -2.265728 & 1.668840 & -1.236444 \\ \mathrm{C} & -1.983866 & 1.769954 & 1.194871 \\ \mathrm{H} & -3.474001 & 0.823204 & 0.171702 \\ \mathrm{H} & -1.604297 & -0.490986 & 0.031551 \\ \mathrm{H} & -2.699272 & 2.675420 & -1.244126 \\ \mathrm{H} & -1.193196 & 1.757365 & -1.424524 \\ \mathrm{H} & -2.398123 & 2.784538 & 1.207971 \\ \mathrm{H} & -0.895391 & 1.841563 & 1.136950 \\ \mathrm{H} & -2.716163 & 1.090738 & -2.045326 \\ \mathrm{H} & -2.251990 & 1.274364 & 2.129805 \\ \mathrm{~N} & -0.962633 & -1.366082 & 0.023456 \\ \mathrm{C} & -1.174017 & -2.144020 & 1.274686 \\ \mathrm{C} & -1.204097 & -2.146975 & -1.220487 \\ \mathrm{H} & 0.001269 & -0.985124 & 0.012083 \\ \mathrm{H} & -2.210632 & -2.476399 & 1.322589 \\ \mathrm{H} & -0.513687 & -3.010939 & 1.287507 \\ \mathrm{H} & -2.241229 & -2.480518 & -1.241960 \\ \mathrm{H} & -0.543251 & -3.013174 & -1.247605 \\ \mathrm{H} & -0.954187 & -1.505888 & 2.129389 \\ ------------------------------------------------\end{array}$

$\left(\mathrm{H}_{2} \mathrm{SO}_{4}\right)_{1} \cdot\left(\mathrm{C}_{3} \mathrm{H}_{6} \mathrm{O}\right)_{1} \cdot\left(\left(\mathrm{CH}_{3}\right)_{2} \mathrm{NH}_{2}\right)_{1}{ }^{+}$

$\begin{array}{llll}\text { C } & 3.808741 & 0.480503 & -0.086259 \\ \text { C } & 4.010945 & -0.016542 & 1.314509 \\ \text { C } & 5.012906 & 0.822717 & -0.905575 \\ \text { O } & 2.685616 & 0.612089 & -0.582142 \\ \text { H } & 5.446734 & 1.751652 & -0.517676 \\ \text { H } & 5.782447 & 0.052943 & -0.807094 \\ \text { H } & 4.739698 & 0.964506 & -1.949320\end{array}$




\begin{tabular}{cccc}
$\mathrm{H}$ & 4.394498 & -1.042175 & 1.262997 \\
$\mathrm{H}$ & 4.772666 & 0.576307 & 1.826101 \\
$\mathrm{H}$ & 3.084343 & -0.016413 & 1.886209 \\
$\mathrm{~N}$ & -3.572723 & 0.527093 & -0.062656 \\
$\mathrm{C}$ & -3.814739 & 1.885852 & 0.513570 \\
$\mathrm{C}$ & -4.804290 & -0.182897 & -0.525158 \\
$\mathrm{H}$ & -2.888953 & 0.590645 & -0.830990 \\
$\mathrm{H}$ & -3.059505 & -0.057897 & 0.621531 \\
$\mathrm{H}$ & -4.279202 & 2.515567 & -0.243401 \\
$\mathrm{H}$ & -4.472402 & 1.790382 & 1.375769 \\
$\mathrm{H}$ & -5.285186 & 0.407669 & -1.303185 \\
$\mathrm{H}$ & -5.478616 & -0.305998 & 0.320513 \\
$\mathrm{H}$ & -2.859960 & 2.310262 & 0.818791 \\
$\mathrm{H}$ & -4.519423 & -1.157318 & -0.917808 \\
$\mathrm{~S}$ & -0.455015 & -0.644789 & 0.051071 \\
$\mathrm{O}$ & -0.963042 & -0.033634 & -1.163759 \\
$\mathrm{O}$ & -1.454909 & -0.952409 & 1.050373 \\
$\mathrm{O}$ & 0.665358 & 0.142993 & 0.710047 \\
$\mathrm{H}$ & 1.557175 & 0.340855 & 0.109709 \\
$\mathrm{O}$ & 0.337557 & -1.975241 & -0.360540 \\
$\mathrm{H}$ & -0.091034 & -2.382204 & -1.129040 \\
\hline---------------------------------------------------
\end{tabular}

$\left(\mathrm{H}_{2} \mathrm{SO}_{4}\right)_{1} \cdot\left(\mathrm{C}_{3} \mathrm{H}_{6} \mathrm{O}\right)_{2} \cdot\left(\left(\mathrm{CH}_{3}\right)_{2} \mathrm{NH}_{2}\right)_{1}{ }^{+}$

$\begin{array}{llll}\text { N } & -2.830805 & 1.662870 & 0.355259 \\ \mathrm{C} & -3.009915 & 2.158282 & 1.750682 \\ \mathrm{C} & -3.567507 & 2.453803 & -0.672812 \\ \mathrm{H} & -1.820499 & 1.666374 & 0.125122 \\ \mathrm{H} & -4.064928 & 2.104449 & 2.014661 \\ \mathrm{H} & -2.428566 & 1.528853 & 2.422346 \\ \mathrm{H} & -2.657671 & 3.186700 & 1.814879 \\ \mathrm{H} & -4.633661 & 2.412297 & -0.456050 \\ \mathrm{H} & -3.217902 & 3.484916 & -0.651967\end{array}$




\begin{tabular}{|c|c|c|c|}
\hline $\mathrm{H}$ & -3.374445 & 2.019304 & -1.652160 \\
\hline $\mathrm{C}$ & 4.987471 & -0.749527 & 0.405522 \\
\hline $\mathrm{C}$ & 6.056831 & -1.696760 & -0.054374 \\
\hline $\mathrm{C}$ & 5.328980 & 0.228738 & 1.495102 \\
\hline $\mathrm{O}$ & 3.873238 & -0.787589 & -0.111811 \\
\hline $\mathrm{H}$ & 5.882307 & -0.263834 & 2.298280 \\
\hline $\mathrm{H}$ & 5.993662 & 0.994496 & 1.079447 \\
\hline $\mathrm{H}$ & 4.439649 & 0.714603 & 1.894229 \\
\hline $\mathrm{H}$ & 7.002061 & -1.170841 & -0.211831 \\
\hline $\mathrm{H}$ & 6.236079 & -2.437392 & 0.733104 \\
\hline $\mathrm{H}$ & 5.747446 & -2.208980 & -0.963381 \\
\hline S & 0.809420 & 0.718978 & -0.583538 \\
\hline $\mathrm{O}$ & -0.097413 & 1.776727 & -0.208379 \\
\hline $\mathrm{O}$ & 0.290829 & -0.599387 & -0.824342 \\
\hline $\mathrm{O}$ & 1.940180 & 0.731178 & 0.468621 \\
\hline $\mathrm{H}$ & 2.722263 & 0.088338 & 0.247965 \\
\hline $\mathrm{O}$ & 1.546300 & 1.212114 & -1.920969 \\
\hline $\mathrm{H}$ & 1.610589 & 0.461267 & -2.530564 \\
\hline $\mathrm{C}$ & -3.964532 & -1.998519 & 0.128115 \\
\hline $\mathrm{C}$ & -5.190917 & -2.871312 & 0.136819 \\
\hline $\mathrm{C}$ & -2.628892 & -2.673020 & -0.043609 \\
\hline $\mathrm{O}$ & -4.068158 & -0.785362 & 0.265005 \\
\hline $\mathrm{H}$ & -6.062133 & -2.301443 & 0.454662 \\
\hline $\mathrm{H}$ & -5.361634 & -3.256739 & -0.874517 \\
\hline $\mathrm{H}$ & -5.046285 & -3.741145 & 0.783078 \\
\hline $\mathrm{H}$ & -2.677832 & -3.444319 & -0.816320 \\
\hline $\mathrm{H}$ & -1.840860 & -1.957640 & -0.276589 \\
\hline $\mathrm{H}$ & -2.377101 & -3.186280 & 0.891823 \\
\hline $\mathrm{H}$ & -3.154556 & 0.671725 & 0.305888 \\
\hline
\end{tabular}

